Meta

Journal des traducteurs

Translators' Journal

\title{
Crise et anticrise
}

\section{Augusto Múrias}

Volume 41, numéro 3, septembre 1996

URI : https://id.erudit.org/iderudit/003682ar

DOI : https://doi.org/10.7202/003682ar

Aller au sommaire du numéro

Éditeur(s)

Les Presses de l'Université de Montréal

ISSN

0026-0452 (imprimé)

1492-1421 (numérique)

Découvrir la revue

Citer cet article

Múrias, A. (1996). Crise et anticrise. Meta, 41(3), 393-404.

https://doi.org/10.7202/003682ar

\section{Résumé de l'article}

L'auteur présente une étude des synonymes du mot crise dans la presse écrite; il traite également les concepts de crise et de solution à la crise et les associations que ce mot suscite universellement, et rend compte d'une manière systématique de Torganisation interne de ces paradigmes, en suggérant d'éventuelles interrelations sémantiques et en mettant en relief les expressions imagées. 


\title{
ÉTUDES TERMINOLOGIQUES ET LINGUISTIQUES
}

\section{CRISE ET ANTICRISE}

\begin{abstract}
Résumé
L'auteur présente une étude des synonymes du mot crise dans la presse écrite; il traite également les concepts de crise et de solution à la crise et les associations que ce mot suscite universellement, et rend compte d'une manière systématique de l'organisation interne de ces paradigmes, en suggérant d'éventuelles interrelations sémantiques et en mettant en relief les expressions imagées.
\end{abstract}

\begin{abstract}
In this study of the word crisis and its synonyms as they are used in print media, the author examines the concepts of crisis and crisis solution as well as the universal associations which they evoke. Providing a systematic description of the internal organization of these paradigms, he suggests possible semantic relationships and highlights figurative expressions.
\end{abstract}

Phénomène dont l'actualité brûlante n'est plus à démontrer, la «crise» ne connaît pas de frontières. Aussi, rien d'étonnant à ce que, la première, la presse, s'en soit emparée avec une belle avidité. Le présent travail se donne pour but d'identifier non seulement les synonymes possibles du mot crise, c'est-à-dire les mots par lesquels il est susceptible d'être remplacé dans un contexte déterminé, mais aussi les associations qu'il suscite universellement. Au demeurant, il n'est pas sans intérêt de constater que les langues européennes paraissent avoir adopté une dénomination dont l'origine, commune, est inspirée de l'ancien grec «krísis»: DE die Krise. DK krisen. EN crisis. ES la crísis. SU krisi. FR la crise. GR кpín. IT la críse. NL de crisis. PT a crise. SV kris. crise:

L'exemple qui suit semble témoigner de la valeur terminologique inhérente au mot

$\mathrm{P} 16.5 .93,342 \quad$ A célula de crise constituída pelo Ministério do Interior, desde quintafeira de manhã, aprovou o plano de acção às $4 h 30$ de sábado.

D'une manière générale, le concept de crise recouvre les informations suivantes: (processus), (instabilité), [préjudiciable (contraire au développement)] (danger) (solution difficile3). Pour compenser la charge négative qui marque l'expression estar em crise, on se prend à espérer qu'il s'agit d'une crise passageira, à laquelle viendra remédier une des innombrables terapias possiveis para a ultrapassar ( $\mathrm{P} 26.5 .93,29)$.

Tout naturellement, des expressions euphémiques ont été créées pour occulter cette charge négative :

$\mathrm{P} 16.5 .93,40 \quad$ «Recessão» ou «crise» são termos que não se encontram na análise do Governo sobre o comportamento da economia, quer nacional, quer comunitária. Fala em «abrandamento» e na influência das economias europeias sobre a portuguesa.

Son emploi se généralisant, le mot crise a bientôt été «vidé», sémantiquement parlant, de son sens:

E 29.5.93, $\mathrm{C1}$ As grandes famillias aristocráticas actuam geralmente do mesmo modo - quando a crise bate à porta e o dinheiro não chega, vendem as jóias.

Meta, XLI, 3, 1996 
Les titres mêmes de la presse écrite semblent parfois exploiter délibérément cette absence de définition sémantique :

P 3.6.93, $52 \quad P S D$ chumba visita à crise -

O PSD resolveu adiar «sine die» uma visita que a Comissão Parlamentar de Economia planeara fazer ao distrito de Leiria e que deveria incidir sobre alguns dos cenários de crise.

En fait, la situation de crise en vient à couvrir une réalité qui peut aller du local au mondial et s'appliquer à tous les aspects de l'activité humaine, aussi précis et limités puissent-ils être par rapport à une crise générale:

$\mathrm{P}$ 10.1.93, 12 EUA anunciam que o Iraque cedeu -

«Crise dos mísseis» acabou.

En dernier ressort, on ne peut attendre d'expressions telles que escapar à crise, fugir à crise ou passar ao lado da crise qu'elles évoquent une réalité marquée par une longévité exceptionnelle. Plus proche de cette réalité et davantage promise à un avenir se trouve sans doute l'expression estar em crise. Sinon, l'exemple suivant serait incompréhensible:

Edos 28.11.92, 1 Obras Públicas à espera da crise.

Conséquence de cette banalisation de l'emploi du mot crise, le relatif «vide» sémantique qui le marque aussi bien dans son essence que dans son impact. Cela est particulièrement patent lorsque le mot est employé d'une manière autonome, c'est-à-dire, que le mot crise ne se trouve pas explicitement associé à un domaine spécifique. Généralement, le contexte linguistique permet cependant d'identifier le domaine en question:

P 17.5.93, 23 Sequestrador de Neuilly estava falido-

(...) Não tinha antecedentes psiquiátricos, apesar de ter sido imediatamente qualificado de "personalidade paranóica» pelo psiquiatra chamado pela policia para acompanhar a crise que se desenrolou durante o sequestro no infantário de Neuilly.

Relevons à ce propos le cas particulier de référence métonymique (par contamination) suivant:

DNng 29.3.93, 9 Promoção e dificuldades da indústria são razóes para a crise hoteleira -

Recessão de cinco estrelas.

Par contre, les exemples suivants témoignent non pas d'un vague phénomène de crise d'une portée globale, mais de l'évolution d'un processus de crise lié à une étape déterminée :

- début

P9.12.92,2 Quem diria, há três anos, que a Europa iria mergulhar numa grave crise económica?

Notons, au passage, le néologisme de la pré-crise, formé par préfixation:

$\mathrm{E} 14.11 .92, \mathrm{C} 2$ Um cenário de pré-crise assola o sector do vidro de embalagem desde o começo do ano.

\section{- évolution}

DNec 23.11.92, 21 Inglaterra : a crise continua.

- dénouement

E 29.5.93, C21 Reino Unido no fim da crise.

En écho à cette pré-crise, on trouve, formé sur le même modèle, le néologisme pós-crise :

I 8.10.92, 2 (...) actualmente nem sequer os indicadores de Agosto se encontram disponiveis, quanto mais os «pós-crise». 
Les particularisations opérées dans le contexte immédiat du mot crise portent habituellement sur les domaines suivants:

(domaine social)

P 16.5.93,66 A crise alentejana não é apenas económica $e$ social. $E$ uma crise moral. Que acentua o tradicional cepticismo do alentejano e o torna ainda mais desconfiado em relaçâo ao futuro.

(ampleur)

P 22.11.92,60 A crise europeia e mundial não chegará com tal intensidade a Portugal.

(durée)

Idinh 25.9.92, 5 (...) o que mantinha a procura interna em prolongada recessão (queda (quantification) de cerca de 1 por cento do PIB prevista para este ano).

E 23.1.93, A24 Clinton: as primeiras crises -

As duas primeiras crises da Administração Clinton ocorreram e foram resolvidas menos de 24 horas após a sua tomada de posse, mas ambas

(localisation dans le temps) prenunciaram o que poderá estar por vir.

Idinh $25.9 .92,6$-Surgiu a crise do dia 16 e as autoridades monetárias espanholas

(intensité / progressivité) tomaram aquela sábia medida de antecipação.

Dans cette rubrique, on distinguera, par ordre de progression, les cas suivants :

- crise + adjectif

I 1.1.93, $26 \quad$ A Rússia é a crise total

- adjectif + crise (l'adjectif se trouve placé devant)

$\mathrm{P} 9.12 .92,3 \quad O$ radicalismo colectivista do Labour perdeu-se na terrível crise dos últimos anos da década de 70.

- crise + adjectif au superlatif

Eec 21.11.92, C16 Mas estamos, também, confrontados com uma crise gravíssima na indústria nacional.

- adjectif au superlatif + crise

P 9.12.92, $5 \quad$ Mesmo a gravíssima crise do aço de que o país era ainda há 20 anos extremamente dependente (...)

À ce titre, on relève d'autres cas fonctionnellement équivalents, où la gradation d'une situation de crise est signalée par un nom ou un adjectif:

P 31.10.92, $15 \quad$ Uma crise em escalada.

On rencontre également des cas où cette progression est inversée, c'est-à-dire où l'intensité de la crise va en s'affaiblissant:

E 14.11.92, A28 (...) desvaloriza a crise e escusa-se a pormenores.

Dans l'analyse de la distribution du mot crise, c'est-à-dire de l'ensemble des contextes où celui-ci peut s'inscrire, on trouve également certaines combinaisons consacrées par l'usage, où le mot crise exerce une fonction attributive, comme sinal de crise, cenário de crise, situação de crise, estado de crise, momento de crise, tempo de crise, gabinete de crise.

Avec ces exemples, on en revient à la question de l'«effacement» sémantique subjacent au mot crise, que vient appuyer par exemple l'association surprenante des deux termes de crise et de récession, habituellement considérés comme sémantiquement voisins :

P 15.5.93,48 Cavaco Silva afirmou que a Europa «tem sido das zonas mais atingidas pela crise de recessãoi. 
E 29.5.93, 8-R $\quad O$ mercado de ilusões nunca fecha $e$ não está em crise, apesar das recessões.

Ce «vide» sémantique est encore manifeste:

- tantôt dans des jeux de mots:

P 10.12.92, $44 \quad$ Sistema desportivo : quadratura de uma crise?

- tantôt dans des expressions familières courantes :

«Isto é que vai uma crise'» (expression employée quand on se trouve confronté à des difficultés d'une certaine gravité);

Não há crise! (expression employée pour nier l'existence d'un problème grave) :

J 30.3.90,35 Há de tudo: desde os [alemães] que falam como nós aos que não dizem uma palavra da nossa língua. Mas nunca há crise. A gente entende-se.

On constate encore ce «vide» sémantique lorsque le mot crise désigne des problèmes concernant l'être humain, et plus concrètement, des problèmes inhérents à une étape de sa croissance (crise de puberdade), à une expérience (crise matrimonial), ou à un symptôme (crise de choro). Fait exception, cependant, l'utilisation du terme en médecine, qui désigne la manifestation aiguë d'un trouble, comme en témoigne l'exemple suivant:

P 21.1.93, 28 Distribuidor dos jogos Nintendo em Portugal vai reforçar recomendaçôes -

Avisos contra as crises [de epilepsia].

Jusqu'ici, nous avons considéré le terme de crise en tant que concept hyperonyme (cover-term, Überbegriff) d'un paradigme lexical formé par des mots (noms, verbes, adjectifs) qui présentent avec lui une affinité sémantique déterminée. Cette affinité tient au fait que les signifiés de ces mots ont entraîné une particularisation sémantique déterminée dans ce terme hyperonyme, incorporé à eux (hyponymes). Cette affinité sémantique se concrétise dans la possibilité de principe que les mots de ce paradigme lexical remplacent, dans un certain contexte, le mot crise. Le titre suivant illustre bien cette capacité de remplacement:

DN 8.5.93, 1 «Crise» autárquica preocupa Cavaco (Página 3).

Et à ladite page 3, on trouve:

Cavaco está descontente com «gestâo» de Fernando Nogueira-

Turbulência autárquica.

Dans le cadre de la présente étude, une distinction essentielle est opérée entre ces mots et expressions qui témoignent d'une situation de crise encore «contrôlable» et ceux qui désignent une situation de crise «extrême», difficilement contrôlable:

DN 8.4.90,4 A crise no partido é, pelo menos por enquanto, controlável.

Idinh 22.1.93, 1 A crise na TAP está incontrolável.

Par la sélection lexicale effectuée, on exclut délibérement des mots et expressions qui ne sont susceptibles que marginalement de laisser entrevoir une situation de crise, comme dans le cas du titre suivant:

Pec 17.5.93, 21 Matrena-Decisão adiada.

Voyons à présent quels peuvent être les paramètres qui permettent de répartir le «corpus» sélectionné au sein du paradigme lexical en question. Il faut souligner que les concepts évoqués, tout comme les relations établies entre eux, sont le fruit d'une présomption tout intuitive et intentionnellement simplifiée. Tout aussi intuitive est la recherche d'un aménagement des mots en fonction d'une progressivité croissante. Pour des raisons évidentes d'économie d'espace, nous nous limitons à reproduire les exemples tirés de la presse faisant référence à des expressions imagées, pour leur force suggestive ${ }^{4}$ : 
«Mésentente» (par opposition à «entente»). On peut classer sous cette rubrique des mots comme desacordo, inconclusivo, conflito (aberto), ainsi que l'expression imagée braço-de-ferro:

P 11.1.92, $40 \quad$ EDP vence braço-de-ferro com afectados do Lindoso.

«Difficultés» (par opposition à «espoir», «faisabilité»). À côté de mots ou d'expressions comme pouco encorajador, delicado, dificuldades, tempos dificeis, contrariar, desencorajador, desfavorável, más notícias, más perspectivas, dar problemas, problemático, em apuros, adverso, negativamente, on trouve:

ESTAR LONGE DE SER UM MAR DE ROSAS

I 1.1.93, 24 Com a oposição à perna e o Banco Mundial nos calcanhares, o futuro NADA SOBRE RODAS do PAIGC está longe de ser um mar de rosas.

Idinh 26.2.93, 15 Nada sobre rodas -

NÃO BATER CERTO

A recuperação do sector [automóvel] chegará em 1994, mas até lá a batalha será dura. Feita modelo a modelo.

Idinh 26.2.93, 28 Nada bate certo na International Business Machines (IBM), a maior empresa de informática a nível mundial.

QUEBRA-CABEÇAS

P 3.1.93, $\quad$ Quebra-cabeças

Mediadores propõem em Genebra plano de pacificação da Bósnia-

PEDRA NO SAPATO Herzegovina.

Pec 4.1.93, 18 Tudo parece muito bem, mas a concertação social é uma pedra no sapato.

COM O PÉ ESQUERDO

DNng 5.4.93, 38 VW inicia ano com pé esquerdo.

REMAR CONTRA A MARÉ

DNng 8,5.93,8 As petrolíferas remaram contra a maré baixa.

ESTAR EM MAUS LENÇÓIS

P 2.10.92, 54 (...) mesmo quando as suas finanças já estão em muito maus lençóis.

ESTAR NA MÓ DE BAIXO

DN 12.1.93, 32 Cinco de Chicago soma terceira derrota em quatro jogos-

Bulls na mó de baixo.

«Incertitude», «menace» (par opposition à «solution», «stabilité»). Peuvent relever de cette rubrique, indefinição, falta de certezas, incerteza, céptico, incógnita, risco, ameaçar, alerta, alarme, ainsi que des mots qui, évoquant l'absence de lumière et de couleur (sombrio, cinzento, negro), suggèrent une perspective incertaine et menaçante, tout comme les expressions métaphoriques suivantes :

CRISE À PORTA

$\mathrm{P} 23.1 .93,1 \quad$ Congresso do CDS com crise à porta.

LUZES VERMELHAS

Idinh 4.6.93, 2 Luzes vermelhas começaram já a acender-se nos ministérios da saúde DEBAIXO DO FOGO

e da Segurança Social.

P 9.12.92, 5 A recente turbulência cambial colocou a sua moeda debaixo de fogo. À BEIRA DO ABISMO

I 1.1.93, $24 \quad$ Nino Vieira parece estar a passar a pior crise de sempre. É a GuinéBissau à beira do abismo. 
«Pessimisme» (par opposition à «espoir»). À classer sous cette rubrique, des mots comme desilusão, pessimismo, resignado, estar condenado, et le néologisme suivant, calqué directement du français:

SINISTROSE

P 31.10.92,25 (...) como enfatizou a televisão suiça num programa especial sobre a crise, um clima de «sinistrose», nas palavras dos suíços-franceses.

«Agitation» (par opposition à «stabilité»). Sous cette rubrique, des mots comme aquecer, instabilidade, nervosismo, confusão, borrasca, turbulência, tensão, ferver, ebulição, sobressalto, reviravolta, tumultuoso, convulsão, em fúria, tempestade, ainsi que les expressions imagées suivantes:

NÃO ACERTAR O PASSO

P 12.5.93, $36 \quad$ Hong Kong ainda não acertou o passo. Ontem voltou a subir depois de ESTAR EM POLVOROSA no dia anterior ter registado perdas importantes nas cotaçôes.

DN 7.5.93, 17 A reestruturação do Ministério da Educação tem também implicações no âmbito laboral e um dos sectores que já está em polvorosa é a Inspecção-Geral de Educação (IGE).

ESTAR EM PÉ DE GUERRA

\section{P 1.11.92, $1 \quad$ Luanda já está em pé de guerra.}

«Malaise» (par opposition à «prospérité»). Rentrent dans cette catégorie des mots comme preocupante, angústia, aflitivo, assustador, traumatizante, doloroso, desesperado, ainsi que:

SOFRER

P 26.6.93, $38 \quad$ Pelo terceiro ano consecutivo, é a imprensa que vai sofrer mais, com uma quebra recorde das receitas publicitárias.

AMARGAR

$\mathrm{DNec} 26.10 .92,3$ A indústria de pasta para papel amarga agora o rescaldo dos anos RESSACA áureos do final da década passada.

$\mathrm{P}$ 10.1.93, $33 \quad$ Investidores de ressaca.

EMPALIDECER

$\operatorname{Pec} 12 / 92$

PESADELO

E 11.1.92, B5 $O$ pesadelo do desemprego.

FANTASMA

P 9.12.92, 2 Em vésperas dos anos 90 e em pleno «boom» provocado, em grande parte, pelo fenómeno psicológico do Mercado Único de 1993, o fantasma da recessão parecia um pesadelo do passado.

«Austérité» (par opposition à «dynamisme», «progrès»). On retrouve ici des mots comme antidespesismo, contençâa, austeridade, rigor, aperto et encore : PÔR TRAVÃO

E 11.1.92, B2 Polónia põe travão.

TRAVAR A FUNDO

Pec 2.11.92, 1 Estagnação desde Abril : Exportaçôes travam a fundo.

«Stagnation», «blocage» (par opposition à «dynamisme», «croissance»). Sous cette rubrique, des mots comme estagnaçäo, estacionário, empatar, paralisação, impasse, marasmo, nulo, bloquear, congelar, cilindrar, entupir, et encore :

BANHO-MARIA

DNng 15.2.93, 23 Construtora em «banho-maria». 
MARCAR PASSO

P 12.1.92, 1 CDS : candidatos marcam passo.

ZERO

DNec 23.11.92, $20 \quad$ Espanha vai ter crescimento zero.

«Carence», «privation» (par opposition à «abondance»). Il faut citer ici des mots et des expressions comme carências, à míngua de, falta, privação et penúria. À relever aussi toute la gamme des mots qui sont liés au régime alimentaire de la dieta forçada:

DIETA

DNec 23.11.92,29 Fiat em dificuldades inicia dieta.

MAGRO

DNng 29.3.93, 30 As receitas são magras, o que terá impedido, segundo Baptista Lopes, APERTAR O CINTO «iniciar a recuperação dos salários».

$\mathrm{P} 23.11 .92,7 \quad$ Cavaco em final de visita a Viseu-

JEJUM Aviso para apertar o cinto.

Jec 10.1.92, $25 \quad O$ jejum forçado dos russos.

EMAGRECIMENTO

$\mathrm{P}$ 14.5.93, $3 \quad O$ que é verdade é que o governador defende que a economia portuguesa deve passar por um periodo de emagrecimento feito à custa de um escudo sobrevalorizado.

TEMPO DE VACAS MAGRAS

P 9.12.92, 4 Prova evidente de que o tempo é de vacas magras é também o drástico FECHAR A TORNEIRA abrandamento dos investimentos.

I 1.1.93, 24 Aquelas duas instituições financeiras certamente agora iräo fechar de vez a torneira.

Une place de choix est réservée dans la querelle opposant les partis politiques en matière de crise au Portugal, à l'opposition «oasis»/«désert», chère à quelques hauts responsables de l'économie nationale:

DESERTO

P 1.12.92, 18 Quando lá fora se fala em crise, enquanto cá dentro se cultiva a imagem do oásis de prosperidade, está-se a ocultar que os maiores desertos de privação, de iniquidade e até de miséria não estão lá fora, mas cá dentro.

«Détérioration» (par opposition à «faisabilité»). Une distinction artificielle est faite ici entre la «détérioration», d'ordre qualitatif, et la «chute», traitée immédiatement après, d'ordre quantitatif. À la notion de «détérioration» s'oppose naturellement celles de «faisabilité» et de «prospérité». Parmi la large gamme des mots qui rentrent dans cette catégorie, on note desaceleração, fragilidade, fraqueza, abalado, agravamento, degradação, deterioração, golpe, falhar, atraso, mediocre, negativo, défice, desequilíbrio, prejuizo, buraco, rombo, estragar, fracasso, desastre, péssimo, fiasco, ainsi que de nombreuses expressions imagées comme:

PERDER TERRENO

P 26.5.93, $3 \quad$ Passou de 39• para 41•

EM DERRAPAGEM

Portugal perde terreno.

P 19.1.93, 40 Portugal crescerá apenas 1,3 por cento em 1993

Economia dos Doze em derrapagem. 
METER ÁGUA

E 11.1.92, B2 CEI já mete água.

NO VERMELHO

Eec 31.12.92, C1 Indústria no «vermelho»-

Um estudo revela que a maior parte das empresas industriais sob a tutela do Estado está àinda em dificuldades.

«Chute» (par opposition à «croissance»). Les exemples sont également très nombreux ici: desaceleração, abrandamento, arrefecimento, contracção, retracção, desgaste, quebra, ficar aquém, depreciação, recuo, redução, retrocesso, diminuição, decréscimo, declínio, descida, baixa, perda, a metade, mínimo. De nouveau, on relève tout un éventail de mots et d'expressions imagés:

FRIO

P 3.6.93, $52 \quad$ Economia mais fria -

PERDER VELOCIDADE

Wall Street iniciou a sessão em queda ligeira.

Pec 4.1.93, 18 (...) ao mesmo tempo que a mesma sociedade corretora, anteriormente líder de mercado, perdeu velocidade, e neste momento é quase

A MEIO GÁS

P 21.11.93, $1 \quad$ Sporting-FC Porto

Campeões a meio gás.

UM PASSO EM FRENTE, DOIS PASSOS ATRÁS

P 12.12.92, 14 Subida do dólar é sinal do agravamento da crise em Angola:

EM QUEDA LIVRE

Um passo em frente, dois passos atrás.

Eec 31.12.92, C1 A Portucel continua em queda livre, face à conjuntura internacional adversa em que o sector das celuloses está mergulhado.

Pour reprendre la distinction déjà opérée auparavant entre crise «contrôlable» et «incontrôlable», nous énumérons ensuite des notions qui évoquent une situation de crise extrême 5 :

«Drame» (pour accentuer la tournure catastrophique des événements et par opposition à «activité»). Ont leur place ici des mots comme drama, dramatismo, desgraça, estado de choque, aterrador, explosivo (adj.) et:

INFERNO

Pec 4.1.93, 18 Juntamente com a Sofin, cairam para os infernos a BCI Valores, outra sociedade envolvida no «caso».

«Faillite» (pour accentuer l'«échec» et par opposition à «opulence»). On peut inclure ici des mots comme ruína, bancarrota, ainsi que les expressions suivantes:

ESTAR A SALDO (cf. aussi estar a saque)

DN 8.4.93,21 Tal situação confirma que o Algarve está a saldo, porque a oferta do FECHAR AS PORTAS imobiliário turístico disparou para níveis muito acima dos desejáveis.

DNng 7.5.93, 9 Cansados de perderem dinheiro - os preços do metal caíram já mais de 53 por cento desde 1985 - muitos produtores fecharam ou vão fechar as suas portas.

«Déroute» (pour accentuer le concept de «détérioration» et par opposition à «succès»). On inclut sous cette rubrique des mots comme ruptura, rotura, desestruturação, descontrolo, colapso, desabamento, descalabro, afundar, catástrofe, sucumbir, incontrolável, inviável, calamidade, barbárie, caos, ainsi que les exemples suivants : 
SEM BÚSSOLA

DNng 7.5.93, 3 Governo está sem bússola.

CAIR A PIQUE

DNng 4.12.92, 12 Bolsa de Hong Kong cai a pique.

BATER NO FUNDO

P 3.6.93, 45 Crise bateu no fundo-

As declarações do primeiro-ministro nipónico, Kiichi Miyazawa, em que se salientava ter a crise que o país atravessa batido no fundo $e$

$O$ COMPLETO ARRASAR que a partir de agora a situação só podia melhorar.

DNng 15.2.93, $21 \mathrm{Na}$ sua opinião, o gigantismo do défice levaria ao arrasar completo das estruturas de apoio ainda existentes nestes sectores.

«Fin» On peut classer dans cette catégorie des termes relativement suggestifs qui laissent entrevoir un danger de mort, comme asfixia, arder, moribundo, decapitação et encore les expressions suivantes :

(ESTAR) NA CORDA BAMBA

P 30.11.93, $56 \quad$ Estação incineradora 'na corda bomba'.

(ESTAR) ENTRE A ESPADA E A PAREDE

I 1.1.93, $24 \quad O$ Governo de Nino Vieira ficou então entre a espada e a parede. (ESTAR) COM A CORDA NA GARGANTA

P 5.11.92, 68 Bombeiros com a corda na garganta.

(SENTIR A) CORDA À VOLTA DO PESCOÇO

Idinh 25.9.92, 5 A Porsche, com 11 milhöes de contos de prejuízos, começa a sentir a corda à volta do pescoço e anunciou uma redução de 25 por cento nos

(col.) DAR O BERRO postos de trabalho.

Pec 4.1.93, 18 A Fnacinvest, essa, deu o berro. Está à espera da nomeação de um administrador judicial, ou a falência.

À l'opposé du paradigme lexical relatif à la crise, il y a celui relatif à l'anticrise (opposition sémantique) :

DNng 5.4.93, 17 Edouard Balladur à frente de uma força de choque anticrise.

Relèvent de ce paradigme des mots qui, par leur sens, laissent entrevoir croissance et prospérité, à l'issue d'une situation de crise. Pour des raisons évidentes d'économie d'espace, on ne reproduira ici que quelques expressions imagées. Cette perspective de croissance et de prospérité est présentée comme une série suivant une courbe ascendante. Les concepts sélectionnés pour illustrer cette rubrique n'ont, évidemment, qu'une valeur indicative :

«Contrôle», «solution» (ce qui suppose donc l'existence précédente d'une situation pénible). Relèvent de cette rubrique des mots comme solução (para a crise), saida, aplacar (a crise), tirar (da crise), ultrapassar, sair, contornar, escapar, salvar, ainsi que l'expression familière suivante:

(col.) SAÍDA DO BECO

P 12.12.92, 11 Ieltsin e o Parlamento russo em confronto - À procura da saída do beco.

«Entente» (par opposition à un climat de «mésentente», de «désaccord»). Signalons dans ce contexte les exemples suivants :

O SIM

I 10.1.92, $1 \quad$ Abecassis e Monteiro à beira do sim.

FAZER AS PAZES

P 10.1.92, $27 \quad$ Bombeiros fazem as pazes com a ARS de Faro. 
«Espoir» (par opposition à «pessimisme»). Sous cette rubrique, les mots esperança, imversão da tendência de evolução negativa, melhoria, melhores dias, reanimado, positivo, boas notícias, boa nova, favorável, promissor, optimismo, ainsi que l'expression imagée suivante:

VER UMA LUZ AO FUNDO DO TÚNEL

P 9.12.92, 5 Depois do pessimismo, a «Central Statistical Office» começa a ver «uma luz ao fundo do túnel».

«Faisabilité» (par opposition à «détérioration»). Pour illustrer cette rubrique, des mots comme desanuviamento, desbloquear, regularizar, bem comportado, rentabilidade, bons resultados, et des expressions comme:

TER O CAMINHO ABERTO

DN 10.1.92, 1 Correios e telefones privados já têm o caminho aberto.

«Dynamisme», «progrès» (par opposition à une tendance à la «stagnation» et au «blocage»). Citons ici les mots mexer, dinamizar, recuperação, retoma, relançamento, valorização, estímulo, incentivo, et encore :

FÔLEGO

P 14.5.93, $3 \quad$ Mede-se pela percepção profunda dos ciclos que a economia vai experimentando, pelas medidas de maior fôlego que toma e pela boa gestão das expectativas que é capaz de fazer.

«Stabilité» (par opposition à une tendance à l'«agitation», à l'«incertitude», à la «menace»). Sous cette rubrique, estabilidade, bem orientado, confiança, tranquilidade, firmeza, consolidado, solidez, seguro, ainsi que l'expression imagée suivante:

$B O A S A U ́ D E$

Idinh 25.9.92, 5 A Ford e a General Motors possuem filiais na Europa que revelam uma boa saúde e lançaram uma série de novos modelos.

«Croissance» (par opposition à une tendance à la «chute»). Parmi les nombreux mots qui relèvent de cette catégorie, on peut citer: acréscimo, aumento, crescimento, alargamento, em alta, multiplicar, ganho, avultado, expansão, progresso, prosperidade, et encore :

GANHAR TERRENO

P 11.5.93, 37 Dólar ganha terreno.

DISPARO

P 12/92, ec $\quad$ Exportações abrandam depois do disparo em 1986.

BOOM

P 9.12.92, 2 Em vésperas dos anos 90 e em pleno «boom» provocado, em grande parte, pelo fenómeno psicológico do Mercado Único de 1993, o

DE VENTO EM POPA fantasma da recessão parecia um pesadelo do passado.

DN 31.1.94 A economia da Noruega vai de vento em popa.

«Essor» (dans le cadre d'une «croissance» et par opposition à une tendance au «malaise»). Sous cette rubrique, on peut citer animação, entusiasmo, festa, fervilhar, et encore :

EUFORIA

$\mathrm{P} 14.12 .92,2 / 3$ Não há razões para euforia na Europa.

FÚRIA

DNng 15.2.92, 21 Há ainda os factores incubados durante a década de 80, que estão na origem das depressões também em curso nos restantes países nórdicos: fúria consumista, liberalização do crédito, especulação nos sectores imobiliário e bolsista. 
LOUCURA

DN 24.12 .92

FRENÉTICO

P $15.5 .93,41$
Diz que a crise neste Natal «leva as pessoas a procurarem cada vez menos peças de roupa», não se tendo notado a loucura natalícia de anos anteriores.

Final frenético-

As aç̧ôes da Bolsa de Madrid terminaram a semana em alta.

«Abondance» (par opposition à «carence», «privation», et pour désigner une croissance soutenue). On peut citer ici abundância, máximo, excedente, et encore les expressions suivantes:

ALARGAR O CINTO (par opposition à apertar o cinto)

P 9.12.92, $4 \quad$ Enquanto os outros apertam o cinto, a Dinamarca anuncia que vai alargá-lo, na esperança de com isso dinamizar o crescimento da economia e lutar contra o desemprego.

ABRIR OS CORDÓES À BOLSA

P 9.12.92, $\quad$ (...) reforçando a teoria de que os britânicos poderáo ter começado a abrir os cordões às suas bolsas.

«Opulence» (par opposition à «faillite»). Dourado et áureo constituent, par excellence, des qualitatifs évoquant l'opulence, tout comme les images suivantes :

OÁSIS (opposé à deserto)

Pec 4.1.93, 18 Já lhes chamaram tudo: dinheiro que veio do céu, presente de Natal e Braga de Macedo terá mesmo visto neles - finalmente - um oásis.

ÉPOCA DE OURO (voir aussi anos de ouro)

P 14.5.93, 40 Mas a época de ouro das pedras nacionais parece estar a chegar ao fim.

«Succès» (par opposition à «déroute»). À côté de sucesso, invejável, recorde, espectacular, coroar, na ribalta, modelo, on peut encore citer les exemples suivants :

SOMA E SEGUE

P 10.1.92, II-6 Finangeste soma e segue.

TER UM LUGAR AO SOL

Pec 4.1.93, 18 (...) uma «holding» que quis ter um lugar ao sol na distribuição, no ar QUADRO DE HONRA condicionado, imprensa, imobiliário, tudo o possível e imaginário.

P 9.12.92, $4 \quad$ Esta taxa de crescimento - uma das mais fortes da CE -, aliada a um fraco défice orçamental, a uma dívida pública que näo excede 48 por cento do PIB e a uma taxa de inflação de 3,1 por cento, coloca a

MILAGRE França no quadro de honra da ortodoxia ecómica da CE.

P9.12.92, 3 Entre 1960 e 80, a média de crescimento da economia britânica situou-se nos 2,3 por cento, para valores que variam entre (...) e os vigorosos 7,7 do «milagre» japonês.

FECHAR COM CHAVE DE OIRO

Pec 4.1.93, 18 Com a aprovação do Fundo de Coesão, na Cimeira de Edimburgo, OURO SOBRE AZUL Cavaco Silva fechou $o$ ano com chave de oiro.

$\mathrm{P}$ 18.5.93, 8 «Ouro sobre azul», no arranque deste «meeting» vermelho na margem sul do Tejo.

Tous ces exemples constituent de multiples concrétisations possibles dans un contexte donné, des concepts de crise et de solution de la crise, lesquelles peuvent d'une manière générale être regroupées selon l'intensité relative de l'apparition du phénomène 
et son «expressivité» (marquée ou neutre). Nous avons cherché à rendre compte d'une manière systématique de l'organisation interne de ces paradigmes, en suggérant d'éventuelles interrelations sémantiques et en mettant en relief les expressions imagées. Sans doute serait-il intéressant de compléter ces considérations par une étude multilingue, qui mettrait en parallèle les dénominations, propres à chaque langue, les plus fréquentes retenues pour ce phénomène d'une portée apparemment mondiale.

AUGUSTO MÚRIAS

Universidade Nova, Lisbonne, Portugal!

Notes

1. Je tiens à remercier M. Pierre Goffin, traducteur au Parlement européen, pour l'adaptation en français.

2. Abréviations utilisées pour désigner les organes de presse portugais cités:

DN Diário de Notícias

ng (suplemento «negócios»)

E Expresso

I O Independente

dinh (suplemento «dinheiro»)

J O Jornal

P OPúblico

ec (suplemento keconomia»)

dos (dossier)

3. Voir la définition du mot crise dans le Grande Dicionário da Língua Portuguesa de J. P. Machado (Amigos do Livro Editores, 1981) : «Situação de um governo cuja conservação encontra dificuldades muito graves».

Ces informations s'appliquent aussi à l'adjectif crítico (dans l'acception de «relatif à une situation de crise»).

4. Pour d'autres exemples, voir le travail du même auteur, intitulé «Sobre a crise», qui sera publié dans un prochain numéro de la revue Terminologie et Traduction (Commission des Communautés européennes).

5. Voir à ce propos les cas déjà mentionnés illustrant la progressivité d'une situation de crise grave (crise gravissima, etc.). 